\title{
Effect of the Educational Package based on Health Belief Model on Nursing Students' Knowledge and Attitude Regarding Human Papillomavirus and Cervical Cancer
}

\author{
Aziza Ebrahim Mahmoud ${ }^{1}$, Samia Abdelhakeem Hasneen Aboud ${ }^{2}$, Eman Mohamed Abd El \\ hakam $^{3}$ and Fatma Kamal Ali ${ }^{4}$
}

(1)B.Sc.Nursing, 2013, (2) Professor of Obstetrics \&Woman's Health Nursing Faculty of Nursing Benha University, (3) Assistant Professor of Obstetrics \&Woman's Health Nursing Faculty of Nursing -Benha University and (4) Lecturer of Woman's Health Nursing Faculty of Nursing -Benha University

\section{Abstract}

Background: Cervical cancer is a significant health problem worldwide. It is the second most common cancer worldwide in women. Human papillomavirus types are the main cause in activating the growth of cervical cancer. This study aimed to evaluate the effect of the educational package based on health belief model on nursing students' knowledge and attitude regarding human papilloma virus and cervical cancer. Design: A quasi-experimental design (pre and posttest) was used for this study. Setting: The study was conducted in faculty of nursing at Benha University. Sample: a purposive sample included (195) female nursing students. Tools of data collection: Three main tools were used, I: A structured self-administered questionnaire which included 3 parts (1) general characteristics of student nurses,(2) medical history (3) knowledge of student nurses regarding human papilloma virus and cervical cancer, II: Modified Likert scale was developed to assess the nursing students' attitudes regarding human papilloma virus and cervical cancer and III: Health Belief Model Scale was adopted to assess the nursing students' beliefs and behaviors regarding human papilloma virus and cervical cancer. Results: There was a high statistically significant improvement among female nursing students regarding their level of knowledge, attitude and Health Belief Model about human papilloma virus and cervical cancer immediate and 4weeks post-intervention compared to pre-intervention with $(\mathrm{P}<0.001)$. Conclusion: Application of the educational package based on health belief model have a positive effect on improving nursing students' knowledge and attitude regarding human papilloma virus and cervical cancer. Recommendations: Application of health belief model for all university students as a healthy preventive behavior towards human papilloma virus and cervical cancer is recommended.

Key words: Educational package, Health belief model, cervical cancer, human papilloma virus.

\section{Introduction}

Cervical cancer is a significant health problem worldwide. It is the second most common cancer worldwide in women. Although cervical cancer is acknowledged as a preventable disease, it is still the major health burden for women in many developing countries because in adequate screening programs. Cervical cancer is a leading cause of death among women in the low and middle-income countries. Approximately half a million new cases of cervical cancer occur globally every year with an estimated $85 \%$ in developing countries and an estimated 270,000 women died of this disease ,where more than $80 \%$ are diagnosed at advanced stage and have poor treatment outcomes (Chan, et al, 2019). 


\section{Effect of the Educational Package based on Health Belief Model on Nursing Students' \\ Knowledge and Attitude Regarding Human Papillomavirus and Cervical Cancer}

Human papillomavirus is a virus with more than 100 subtypes identified, including nearly 40 that may directly infect the genitals. There are a few high-risk types such as types 16 and 18, which may produce cervical cell abnormalities, and these types are precursors to oral and genital carcinomas. The risk of human papillomavirus related cancers remains high in individuals unvaccinated against HPV. Human Papillomavirus vaccination is an effective approach for primary prevention of cervical cancer and presents an opportunity to reduce the burden from cervical cancer in a number of countries (Hampson, et al, 2021).

The educational package is developed to allow the learner to be free to select what, how, when and where to learn. This flexibility is an importance characteristic in open learning process. The learner becomes accustomed more and more to non-formal mode of education (Ahmed, et al, 2018).

Using appropriate models and theories is the first step in the process of programming for health education. Based on the different studies results, the HBM is an appropriate educational model; It is a comprehensive model that rather than controlling the disease, is mostly used for its prevention and emphasizes on how one's perceptions cause motivation and movement, and lead to behavior, According to the HBM, health behavior is the result of a series of central beliefs of people concerning their conceptions of perceived personal susceptibility, perceived severity of the disease, perceived benefits of the new behavior, perceived barriers for applying a preventive health behaviors ,cues to action, and perceived self-efficacy to perform preventive behaviors (Eghbal,et al,2021).
The nurse is the cornerstone in avoidance of cervical cancer owing to being a vital $\operatorname{cog}$ in the healthcare delivery system. Fortunately, the nurse plays a key role in health education and promotion. Moreover, the nurse has an important task of imparting information on risk factors, discovering early signs of cervical cancer, HPV, and encouraging females to undergo cervical cancer screening frequently (Maver and Poljak, 2019)

\section{Significance of the study}

Egypt has a population of 30.55 million, where women aged 19 years and older are at a risk of developing cervical cancer. Recent estimations indicate that every year 866 females are diagnosed with cervical cancer and 373 die owing to it. In Egypt, cervical cancer ranks as the 10th most frequent cancer among women between 19 and 44 years of age. Early detection is critically important in reducing deaths owing to cervical cancer (Ampofo, et al, 2020)

\section{Aim of the study}

This study aimed to evaluate the effect of the educational package based on health belief model on nursing students' knowledge and attitude regarding human papilloma virus and cervical cancer.

\section{Study hypothesis:}

Nursing students who referred the educational package based on health belief model were shown a significant improvement in their knowledge and attitude regarding human papilloma virus and cervical cancer post intervention compared to pre intervention.

\section{Subjects and method}

\section{Study Design:}

A quasi-experimental (an intervention study) pre and posttest. 


\section{Study setting:}

This study was conducted at faculty of nursing, Banha University.

\section{Sample:}

Sample type: A Purposive sample was selected from the above mentioned study setting.

Sample size: The total number of study sample was (195) female students and was chosen according to this formula: $\mathrm{N}=\frac{\mathrm{N}}{1+\mathrm{N}(\mathrm{e}) 2}$ Where:

$\mathrm{N}=$ total female nursing students number (380). $\quad \mathrm{e}=$ margin error (0.05).

\section{Inclusion criteria:}

- Female nursing students at the first academic year from faculty of nursing.

\section{Exclusion criteria:}

- Male nursing students.

\section{Tools for data collection:}

\section{Tool I: A structured self-administered} questionnaire:

It was constructed by the researcher after reviewing a related literature as (Ahmed, et al 2018; Naz, et al, 2018) and under the guidance of supervisors it was translated into Arabic language. It was included the following 3 parts:

Part (1): Assessment of nursing students' general characteristics such as (age, place of residence, marital status, special courses on HPV and cervical cancer, source of information)

Part (2): Medical history such as (previous history of cervical cancer, previous medical history of any type of cancer, any family member had a previous history of cervical cancer or HPV).

Part (3): Assessment of nursing student's knowledge regarding HPV and cervical cancer.it consisted of 4sections:

Section (1) was concerned with knowledge related to cervical cancer, it consisted of (7) items.
Section (2) was concerned with knowledge related to PAP smear test, it consisted of (4) items.

Section (3) was concerned with knowledge related to human papilloma virus infection, it consisted of (8) items.

Section (4) was concerned with knowledge related to HPV vaccine, it consisted of (9) items.

\section{Scoring system for nursing students' knowledge}

All knowledge variables were weighted according to items included in each question. Each item was given a score (2) when the answer was complete correct answer, a score (1) when the answer was incomplete correct and score (0) when the answer was I don't know. The total score of each section was calculated by summation of its items the total score for the knowledge of each student was calculated by the addition of total scores of all sections.

Students' total knowledge score was converted into total percent and graded as the following:

- Good: ( $\geq 75 \%$ correct answers).

- Average: (60 - < 75\% correct answers).

- Poor: ( $<60 \%$ correct answers).

Tool II: - Modified likert scale for female Nursing students' attitude:-

It was developed by the researcher after reviewing a related literature as (Dönmez, et al, 2019) to measure the female nursing students' attitude regarding HPV and cervical cancer. It consisted of 19 statements.

Scoring system for nursing students' attitude scale

To obtain the outcome of attitude scale, each statement was scored as following: (2) if the response was "agree", (1) if it was "sometimes" and (Zero) if it was "disagree". The total score was 


\section{Effect of the Educational Package based on Health Belief Model on Nursing Students' \\ Knowledge and Attitude Regarding Human Papillomavirus and Cervical Cancer}

expressed as a percentage. The total score of attitude was classified into:

- $\quad$ Positive attitude: $\geq 75 \%$

- Negative attitude: $<75 \%$

Tool III: -Health Belief Model Scale (HBM scale):-

The HBM was adapted from (Ampofo,et al,2020; Annan,et al,2019) Modification was done by the researcher and under the guidance of supervisors it was translated into Arabic language. It composed of 43 items including 6 subscales (perceived susceptibility -seven items, perceived severity to cervical cancer -seven items, perceived barriers eleven items, perceived benefits -six items, cues to action -seven items, selfefficacy -five items).

\section{Scoring System of Health Belief} Model: The questionnaire was include 43 items on a3 point Likert scale (agree, sometimes, disagree) To obtain the outcome of HBM scale, each statement was scored as following: (2) if the response was "agree", (1) if it was "sometimes" and (Zero) if it was "disagree" in subscales of benefits, cues to action, self-efficacy.

The score reversed to negative points in subscale of perceived barriers; each statement was scored as following: (2) if the response was "dis agree", (1) if it was "sometimes" and (Zero) if it was "agree".

The total score was expressed as a percentage. The total score of HBM was classified into:

-Positive behavior: $\geq 75 \%$

-Negative behavior: $<75 \%$

\section{Validity of the tools:}

Tools of data collection were reviewed by panel expertise (three specialized university professionals in the field of obstetrics and gynecological nursing .Minor modification was done based on jury opinion.

\section{Reliability of the tools:}

The reliability was done by Cronbach's Alpha coefficient test which revealed that: the internal consistency of knowledge assessment questionnaire was 0.81 , the internal consistency of attitude assessment scale was 0.79 and the internal consistency of HBM scale was 0.76 .

\section{Ethical consideration:}

- $\quad$ The aim of the study was explained to each student nurse before applying the tools to gain their confidence and trust.

- $\quad$ Ethical aspect was considered before starting the study that includes the following:

- The research approval was obtained from Scientific Research Ethical Committee, Faculty of Nursing at Benha University before starting the study.

- An oral consent was obtained from each student nurse to participate in the study and withdraw when she needs without obligation.

- The study has no physical, social, or psychological risk on the Participants; the data were collected and treated confidentially.

\section{Pilot study}

The pilot study was conducted on $10 \%$ (19 female nursing students) of the total sample to test the clarity, arrangement, feasibility and applicability of the tools as well as the estimation of the time needed to fill the questions and to make sure that the items were understood. No modifications were done and pilot sample was included in the study sample.

\section{Field work:}

The researcher was attended the previous mentioned study setting for two days 
per week (from 9 am to $4 \mathrm{pm}$ ). After taking the legal aspect of ethics in research.

The current study started from the beginning of first November 2020 to the end of December 2020, covering a long period of 2 months, the researcher was provided appropriate place for interviewing the student nurses

Students were classified into 10 subgroups (each group involved approximately 20 students); the researcher start the sessions at the end of clinical area, the researcher was interviewed three groups of student nurses/day.

The researcher introduced herself, greet students, the purpose of the study was explained by the researcher and an oral consent was taken to participate in the study.

The researcher distributed a structured self-administered questionnaire to students (tool no. I) (part 1and2) to assess student's general characteristics، medical history; Part 3 (pre-post test) was used to assess knowledge regarding HPV and cervical cancer.

Modified likert scale (tool no. II) (Prepost test) was used to assess female nursing students' attitude regarding HPV and cervical cancer.

Finally, Health belief model scale (tool no. III) (Pre-post test) was used to assess female nursing students' beliefs and behaviors regarding HPV and cervical cancer.

The educational program included five interactive sessions "twice per week" for two consecutive weeks, for each group of students. Each session was conducted for (30minutes); the feedback was given in the beginning of each session about the previous one

The content of sessions was as following: First session: it included information regarding cervical cancer .Second session: it included information regarding pap smear test. Third session: it included information regarding HPV infection. Fourth session: it included information regarding HPV vaccination and Fifth session: was concerned with explanation of health belief model and its role to make people adopt disease prevention strategies or screening tests for early detection of diseases.

Methods of teaching were lecture, group discussion, role play, brain storming were used. Media were (lab-top computer, figures, flip chart as well as audiovisual aids (data show presentation) was used as a visual aid to help in clarifying the presented knowledge. During and after the presentation the researcher was encouraged the active participation of the student nurses by asking questions and receiving feedback. Distribution of the educational booklet that contains (all the updated information regarding cervical cancer and HPV with illustrated images) to all students.

\section{Limitation of the Study}

Sometimes it was difficult to assemble the entire group for the educational sessions because of their different times of their practical sections.

\section{Statistical analysis:}

Data were verified prior to computerized entry. The Statistical Package for Social Sciences (SPSS version 20.0) was used. Descriptive statistics were applied (e.g., mean, standard deviation, frequency and percentages). Test of significance (chi square) was applied to test the study hypothesis.

\section{Results}

Table (1) clarifies general characteristics of the studied sample. It was clear that about more than three quarters (76.9\%) of studied sample were in age group 18 years with a mean age of $17.86 \pm 0.51$ years. Regarding the residence, about three 


\section{Effect of the Educational Package based on Health Belief Model on Nursing Students' \\ Knowledge and Attitude Regarding Human Papillomavirus and Cervical Cancer}

quarters $(74.4 \%)$ of them lived in rural areas. Furthermore, the majority of them $(97.4 \%)$ were single. Moreover, all studied sample (100.0\%) didn't attend any scientific seminars or conferences on cervical cancer or human papilloma virus .

Table (2) showed that, there was a marked improvement regarding all items of studied sample total knowledge (knowledge about cervical cancer, pap smear test, human papillomavirus infection, and human papillomavirus vaccine) post implementation of the educational package based on health belief model with a highly statistical significant difference $(p<0.001)$ between pre ,immediate post, and four weeks post intervention.

Figure (1) displays that, (13.3\%), $(82.1 \%)$ and $(78.4 \%)$ of studied sample had good total knowledge score at preintervention, immediate post-intervention and four weeks post intervention phases respectively. While, it was revealed that (79.5\%), (11.7\%) and (14.8\%)of studied sample had poor total knowledge score at preintervention, immediate post-intervention and four weeks post intervention phases respectively.

Table (3) showed that, there was a marked improvement in all items of studied sample attitude regarding cervical cancer and PAP test post implementation of the educational package based on health belief model with a highly statistical significant difference $(p<0.001)$ between pre ,immediate post, and four weeks post intervention.

Table (4) showed that, there was a marked improvement in all items of studied sample attitude regarding human papillomavirus and its vaccination post implementation of the educational package based on health belief model with a highly statistical significant difference $(\mathrm{p}<0.001)$ between pre ,immediate post, and four weeks post intervention.

Figure (2) showed that, (21.0\%), $(74.4 \%)$ and $(72.8 \%)$ of studied sample had positive total attitude score at pre-intervention ,immediate post-intervention and four weeks post intervention phases respectively. On the other hand, it was revealed that $(79.0 \%)$, $(25.6 \%)$ and $(27.2 \%)$ of studied sample had negative total attitude score at preintervention ,immediate post-intervention and four weeks post intervention phases respectively.

Table (5) showed that, there was a marked improvement in all items of studied sample health belief model (total Perceived susceptibility, total Perceived severity, total perceived benefits, total Perceived barriers, total Cues to action and total Perceived selfefficacy) post intervention with a highly statistical significant difference $(\mathrm{p}<0.001)$ between pre, immediate post intervention, and four weeks post intervention.

Figure (3) showed that, (19.0\%) ,(67.2\%) and(65.2\%) of studied sample had positive total HBM score at pre-intervention , immediate post intervention and 4 weeks post intervention phases respectively. On the other hand, it was revealed that $(81.0 \%),(32.8 \%)$ and $(34.4 \%)$ of studied sample had negative total HBM score at pre-intervention ,immediate post-intervention and four weeks post intervention phases respectively.

Table (6) clarifies that there was a highly positive statistical correlation between total knowledge, total attitude and total HBM scores regarding cervical cancer and human papilloma virus at pre and immediate post intervention and four weeks post-intervention phases $(\mathrm{p}<0.001)$. 
Table (1): Frequency Distribution of the studied sample according to their general characteristics $(\mathbf{n}=195)$.

\begin{tabular}{|c|c|c|}
\hline General characteristics & No & $\%$ \\
\hline \multicolumn{3}{|l|}{ Age } \\
\hline 17 & 37 & 19 \\
\hline 18 & 150 & 76.9 \\
\hline 19 & 5 & 2.6 \\
\hline$\geq 20$ & 3 & 1.5 \\
\hline Mean \pm SD & \multicolumn{2}{|c|}{$17.86 \pm 0.51$} \\
\hline \multicolumn{3}{|l|}{ Residence } \\
\hline Urban & 50 & 25.6 \\
\hline Rural & 145 & 74.4 \\
\hline \multicolumn{3}{|l|}{ Marital status } \\
\hline Single & 190 & 97.4 \\
\hline Married & 5 & 2.6 \\
\hline \multicolumn{3}{|c|}{$\begin{array}{l}\text { Attended any scientific seminars or conferences on cervical cancer or human } \\
\text { papilloma virus }\end{array}$} \\
\hline Yes & 0 & 0.0 \\
\hline No & 195 & 100.0 \\
\hline
\end{tabular}

Table (2): Frequency Distribution of studied sample regarding their total knowledge score about cervical cancer

\begin{tabular}{|c|c|c|c|c|c|c|c|c|}
\hline \multirow{2}{*}{$\begin{array}{l}\text { Knowledge } \\
\text { items }\end{array}$} & \multicolumn{2}{|c|}{ Pre-intervention } & \multicolumn{2}{|c|}{$\begin{array}{c}\text { Immediate post- } \\
\text { intervention }\end{array}$} & \multicolumn{2}{|c|}{$\begin{array}{l}4 \text { weeks post- } \\
\text { intervention }\end{array}$} & \multicolumn{2}{|c|}{ Friedman test } \\
\hline & No & $\%$ & No & $\%$ & No & $\%$ & $\mathbf{X} 2$ & P-value \\
\hline \multicolumn{9}{|c|}{ Knowledge about cervical cancer } \\
\hline Good & 25 & 12.8 & 156 & 80.0 & 159 & 81.5 & \multirow[t]{3}{*}{290.2} & \multirow[t]{3}{*}{0.000} \\
\hline Average & 22 & 11.3 & 20 & 10.3 & 12 & 6.2 & & \\
\hline Poor & 148 & 75.9 & 19 & 9.7 & 24 & 12.3 & & \\
\hline \multicolumn{9}{|c|}{ Knowledge about the human papillomavirus } \\
\hline Good & 31 & 15.9 & 167 & 85.6 & 164 & 84.1 & \multirow[t]{3}{*}{280.3} & \multirow[t]{3}{*}{0.000} \\
\hline Average & 16 & 8.2 & 8 & 4.1 & 8 & 4.1 & & \\
\hline Poor & 148 & 75.9 & 20 & 10.3 & 24 & 11.8 & & \\
\hline \multicolumn{9}{|c|}{ Knowledge about Pap smear test } \\
\hline Good & 24 & 12.3 & 149 & 76.4 & 145 & 74.4 & \multirow[t]{3}{*}{287.6} & \multirow[t]{3}{*}{0.000} \\
\hline Average & 11 & 5.6 & 22 & 11.3 & 24 & 12.3 & & \\
\hline Poor & 160 & 82.1 & 24 & 12.3 & 26 & 13.3 & & \\
\hline \multicolumn{9}{|c|}{ Knowledge about human papillomavirus vaccine } \\
\hline Good & 26 & 13.4 & 161 & 82.6 & 156 & 80.0 & \multirow[t]{3}{*}{291.3} & \multirow[t]{3}{*}{0.000} \\
\hline Average & 18 & 9.2 & 16 & 8.2 & 17 & 8.7 & & \\
\hline Poor & 151 & 77.4 & 18 & 9.2 & 22 & 11.3 & & \\
\hline
\end{tabular}


Effect of the Educational Package based on Health Belief Model on Nursing Students' Knowledge and Attitude Regarding Human Papillomavirus and Cervical Cancer

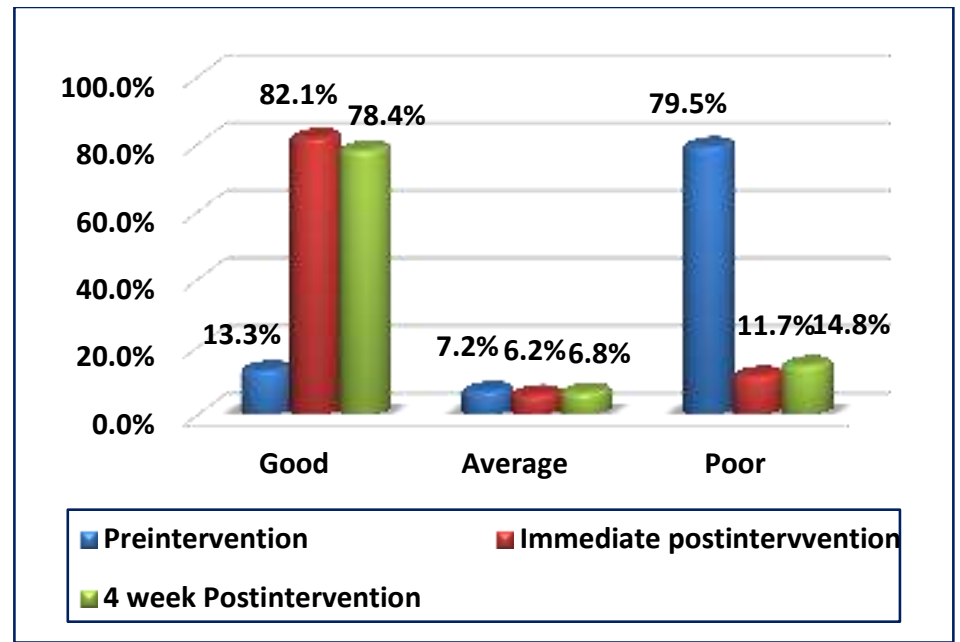

Figure (1): Percentage distribution of studied sample regarding their total knowledge score at pre, immediate post intervention and 4 weeks post intervention phases $(n=195)$ 


\section{$\underline{\text { Aziza Ebrahim, Samia Abdelhakeem, Eman Abd El hakam and Fatma Kamal }}$}

Table (3): Frequency Distribution of studied sample regarding their attitude about cervical cancer and PAP test at Pre, immediate -post intervention and 4weeks Post- intervention phases (n=195).

\begin{tabular}{|c|c|c|c|c|c|c|c|c|c|c|c|c|c|c|c|c|c|c|c|c|}
\hline \multirow{3}{*}{ Attitudes items } & \multicolumn{6}{|c|}{ Pre-intervention } & \multicolumn{6}{|c|}{ Immediate Post-intervention } & \multicolumn{6}{|c|}{4 weeks post-intervention } & \multicolumn{2}{|c|}{$\begin{array}{l}\text { Friedman } \\
\text { test }\end{array}$} \\
\hline & \multicolumn{2}{|c|}{ Agree } & \multicolumn{2}{|c|}{ Sometimes } & \multicolumn{2}{|c|}{ Disagree } & \multicolumn{2}{|c|}{ Agree } & \multicolumn{2}{|c|}{ Sometimes } & \multicolumn{2}{|c|}{ Disagree } & \multicolumn{2}{|c|}{ Agree } & \multicolumn{2}{|c|}{ Sometimes } & \multicolumn{2}{|c|}{ Disagree } & \multirow[b]{2}{*}{$\mathbf{X} 2$} & \multirow{2}{*}{$\begin{array}{c}\text { P- } \\
\text { value }\end{array}$} \\
\hline & No & $\%$ & No & $\%$ & No & $\%$ & No & $\%$ & No & $\%$ & No & $\%$ & No & $\%$ & No & $\%$ & No & $\%$ & & \\
\hline $\begin{array}{l}\text { Cervical cancer is a } \\
\text { preventable disease }\end{array}$ & 31 & 15.9 & 45 & 23.1 & 119 & 61.0 & 145 & 74.4 & 27 & 13.8 & 23 & 11.8 & 141 & 72.3 & 34 & 17.4 & 20 & 10.3 & 279.4 & 0.000 \\
\hline $\begin{array}{l}\text { Cervical cancer can be } \\
\text { detected early by (PAP } \\
\text { test). }\end{array}$ & 40 & 20.5 & 53 & 27.2 & 102 & 52.3 & 152 & 77.9 & 23 & 11.8 & 20 & 10.3 & 149 & 76.4 & 28 & 14.4 & 18 & 9.2 & 268.0 & 0.000 \\
\hline $\begin{array}{l}\text { Cervical cancer may } \\
\text { affect a girl who has a } \\
\text { positive history of the } \\
\text { disease. }\end{array}$ & 57 & 29.2 & 67 & 34.4 & 71 & 36.4 & 160 & 82.0 & 20 & 10.3 & 15 & 7.7 & 161 & 82.6 & 25 & 12.8 & 9 & 4.6 & 246.0 & 0.000 \\
\hline $\begin{array}{l}\text { The most common } \\
\text { category of cervical } \\
\text { cancer is women with } \\
\text { multiple sexual relations } \\
\text { and who are married at a } \\
\text { young age }\end{array}$ & 39 & 20 & 44 & 22.6 & 112 & 57.4 & 153 & 78.4 & 22 & 11.3 & 20 & 10.3 & 152 & 77.9 & 23 & 11.8 & 20 & 10.3 & 271.3 & 0.000 \\
\hline $\begin{array}{l}\text { The "PAP" screening } \\
\text { test is very important to } \\
\text { detect any change in } \\
\text { cervical cells or } \\
\text { infection with HPV }\end{array}$ & 41 & 21.0 & 56 & 28.7 & 98 & 50.3 & 149 & 76.4 & 28 & 14.4 & 18 & 9.2 & 153 & 78.5 & 25 & 12.8 & 17 & 8.7 & 269.5 & 0.000 \\
\hline $\begin{array}{l}\text { Failure to perform the } \\
\text { "PAP" screening test }\end{array}$ & 65 & 33.3 & 73 & 37.4 & 57 & 29.3 & 153 & 78.4 & 22 & 11.3 & 20 & 10.3 & 149 & 76.4 & 24 & 12.3 & 22 & 11.3 & 213.7 & 0.000 \\
\hline
\end{tabular}




\section{Effect of the Educational Package based on Health Belief Model on Nursing Students' Knowledge and Attitude Regarding Human}

Papillomavirus and Cervical Cancer

\begin{tabular}{|c|c|c|c|c|c|c|c|c|c|c|c|c|c|c|c|c|c|c|c|c|}
\hline $\begin{array}{l}\text { leads to bad results due } \\
\text { to the difficulty of early } \\
\text { detection of cervical } \\
\text { cancer }\end{array}$ & & & & & & & & & & & & & & & & & & & & \\
\hline $\begin{array}{l}\text { One of the reasons } \\
\text { women do not have a } \\
\text { cervical examination is } \\
\text { fear of the test results }\end{array}$ & 84 & 43.1 & 65 & 33.3 & 46 & 23.6 & 138 & 70.8 & 35 & 17.9 & 22 & 11.3 & 142 & 72.8 & 33 & 16.9 & 20 & 10.3 & 291.3 & 0.000 \\
\hline $\begin{array}{l}\text { The examination test } \\
\text { "PAP" is inexpensive, } \\
\text { painless, and takes a few } \\
\text { minutes }\end{array}$ & 29 & 14.9 & 37 & 18.9 & 129 & 66.2 & 141 & 72.3 & 34 & 17.4 & 20 & 10.3 & 146 & 74.9 & 29 & 14.8 & 20 & 10.3 & 158.2 & 0.000 \\
\hline $\begin{array}{l}\text { The examination test } \\
\text { "PAP" is very important } \\
\text { for any married woman } \\
\text { from the age of } 21 \\
\text { years. }\end{array}$ & 42 & 21.5 & 51 & 26.2 & 102 & 52.3 & 139 & 71.3 & 33 & 16.9 & 23 & 11.8 & 138 & 70.8 & 35 & 17.9 & 22 & 11.3 & 259.3 & 0.000 \\
\hline
\end{tabular}




\section{$\underline{\text { Aziza Ebrahim, Samia Abdelhakeem, Eman Abd El hakam and Fatma Kamal }}$}

Table (4): Frequency Distribution of studied sample regarding their attitude about human papillomavirus and its vaccination at Pre, immediate -post intervention and 4weeks Post- intervention phases ( $n=195)$.

\begin{tabular}{|c|c|c|c|c|c|c|c|c|c|c|c|c|c|c|c|c|c|c|c|c|}
\hline \multirow{3}{*}{ Attitudes items } & \multicolumn{6}{|c|}{ Pre-intervention } & \multicolumn{6}{|c|}{ Immediate Post-intervention } & \multicolumn{6}{|c|}{4 weeks post-intervention } & \multicolumn{2}{|c|}{$\begin{array}{c}\text { Friedman } \\
\text { test }\end{array}$} \\
\hline & \multicolumn{2}{|c|}{ Agree } & \multicolumn{2}{|c|}{$\begin{array}{c}\text { Sometime } \\
\text { s }\end{array}$} & \multicolumn{2}{|c|}{ Disagree } & \multicolumn{2}{|c|}{ Agree } & \multicolumn{2}{|c|}{$\begin{array}{c}\text { Sometime } \\
\text { s }\end{array}$} & \multicolumn{2}{|c|}{ Disagree } & \multicolumn{2}{|c|}{ Agree } & \multicolumn{2}{|c|}{$\begin{array}{c}\text { Sometime } \\
\text { s }\end{array}$} & \multicolumn{2}{|c|}{ Disagree } & \multirow[t]{2}{*}{$\mathrm{X} 2$} & \multirow{2}{*}{$\begin{array}{c}\text { P- } \\
\text { valu } \\
\text { e }\end{array}$} \\
\hline & No & $\%$ & No & $\%$ & No & $\%$ & No & $\%$ & No & $\%$ & No & $\%$ & No & $\%$ & No & No & $\%$ & No & & \\
\hline $\begin{array}{l}\text { Infection with the HPV } \\
\text { is one of the main } \\
\text { causes of cervical } \\
\text { cancer }\end{array}$ & 15 & 7.7 & 36 & 18.5 & $\begin{array}{c}14 \\
4\end{array}$ & 73.8 & 142 & 72.8 & 33 & 16.9 & 20 & 10.3 & $\begin{array}{l}14 \\
6\end{array}$ & 74.9 & 29 & 14.8 & 20 & 10.3 & $\begin{array}{l}317 . \\
4\end{array}$ & $\begin{array}{l}0.00 \\
0\end{array}$ \\
\hline $\begin{array}{l}\text { Sexual contact is not } \\
\text { the only way to } \\
\text { transmit HPV infection }\end{array}$ & 25 & 12.8 & 39 & 20.0 & $\begin{array}{c}13 \\
1\end{array}$ & 67.2 & 133 & 68.2 & 34 & 17.4 & 28 & 14.4 & 132 & 67.7 & 33 & 16.9 & 30 & 15.4 & $\begin{array}{l}279 . \\
4\end{array}$ & $\begin{array}{l}0.00 \\
0\end{array}$ \\
\hline $\begin{array}{llr}\text { Both women } & \text { and } & \text { men } \\
\text { are at risk } & \text { of } \\
\text { contracting HPV } & \\
\end{array}$ & 33 & 16.9 & 43 & 22.1 & $\begin{array}{c}11 \\
9\end{array}$ & 61.0 & 152 & 77.9 & 23 & 11.8 & 20 & 10.3 & 153 & 78.5 & 25 & 12.8 & 17 & 8.7 & $\begin{array}{l}283 . \\
5\end{array}$ & $\begin{array}{l}0.00 \\
0\end{array}$ \\
\hline $\begin{array}{l}\text { Multiple sexual } \\
\text { partners increase the } \\
\text { chances of contracting } \\
\text { HPV }\end{array}$ & 42 & 21.6 & 50 & 25.6 & $\begin{array}{c}10 \\
3\end{array}$ & 52.8 & 149 & 76.4 & 24 & 12.3 & 22 & 11.3 & $\begin{array}{l}14 \\
6\end{array}$ & 74.9 & 29 & 14.8 & 20 & 10.3 & $\begin{array}{l}260 . \\
0\end{array}$ & $\begin{array}{l}0.00 \\
0\end{array}$ \\
\hline $\begin{array}{l}\text { Vaccination against } \\
\mathrm{HPV} \text { is safe and } \\
\text { effective. }\end{array}$ & 32 & 16.4 & 28 & 14.4 & $\begin{array}{c}13 \\
5\end{array}$ & 69.2 & 132 & 67.7 & 33 & 16.9 & 30 & 15.4 & 135 & 69.2 & 34 & 17.4 & 26 & 13.4 & $\begin{array}{l}264 . \\
5\end{array}$ & $\begin{array}{l}0.00 \\
0\end{array}$ \\
\hline $\begin{array}{l}\text { It is better to take a } \\
\text { vaccination against } \\
\text { HPV than to do "PAP" } \\
\text { test every year. }\end{array}$ & 29 & 14.9 & 34 & 17.4 & $\begin{array}{c}13 \\
2\end{array}$ & 67.7 & 135 & 69.2 & 34 & 17.4 & 26 & 13.4 & 138 & 70.8 & 35 & 17.9 & 22 & 11.3 & $\begin{array}{l}278 . \\
4\end{array}$ & $\begin{array}{l}0.00 \\
0\end{array}$ \\
\hline Taking a vaccine & 41 & 21.0 & 52 & 26.7 & 10 & 52.3 & 138 & 70.8 & 32 & 16.4 & 25 & 12.8 & 141 & 72.3 & 36 & 18.5 & 18 & 9.2 & 257. & 0.00 \\
\hline
\end{tabular}


Papillomavirus and Cervical Cancer

\begin{tabular}{|c|c|c|c|c|c|c|c|c|c|c|c|c|c|c|c|c|c|c|c|c|}
\hline $\begin{array}{lrr}\text { against the } & \text { HPV } \\
\text { protects } & & \text { against } \\
\text { cervical cancer. } & \end{array}$ & & & & & 2 & & & & & & & & & & & & & & 1 & 0 \\
\hline $\begin{array}{l}\text { Vaccination against } \\
\text { HPV should be added } \\
\text { to the compulsory } \\
\text { vaccination schedule by } \\
\text { the Ministry of Health } \\
\text { for males and females. }\end{array}$ & 46 & 23.5 & 59 & 30.3 & 90 & 46.2 & 146 & 74.9 & 29 & 14.8 & 20 & 10.3 & 146 & 74.9 & 29 & 14.8 & 20 & 10.3 & $\begin{array}{l}258 . \\
0\end{array}$ & $\begin{array}{l}0.00 \\
0\end{array}$ \\
\hline $\begin{array}{l}\text { Awareness about } \\
\text { cervical cancer and } \\
\text { HPV is very important } \\
\text { for males and females. }\end{array}$ & 63 & 32.3 & 54 & 27.7 & 78 & 40.0 & 141 & 72.3 & 36 & 18.5 & 18 & 9.2 & 142 & 72.8 & 33 & 16.9 & 20 & 10.3 & $\begin{array}{l}223 . \\
3\end{array}$ & $\begin{array}{l}0.00 \\
0\end{array}$ \\
\hline $\begin{array}{l}\text { I would like to receive } \\
\text { vaccination against } \\
\text { human papillomavirus }\end{array}$ & 15 & 7.8 & 37 & 18.9 & $\begin{array}{c}14 \\
3\end{array}$ & 73.3 & 140 & 71.8 & 30 & 15.4 & 25 & 12.8 & $\begin{array}{l}14 \\
6\end{array}$ & 74.9 & 29 & 14.8 & 20 & 10.3 & $\begin{array}{r}307 . \\
0\end{array}$ & $\begin{array}{r}0.00 \\
0\end{array}$ \\
\hline
\end{tabular}




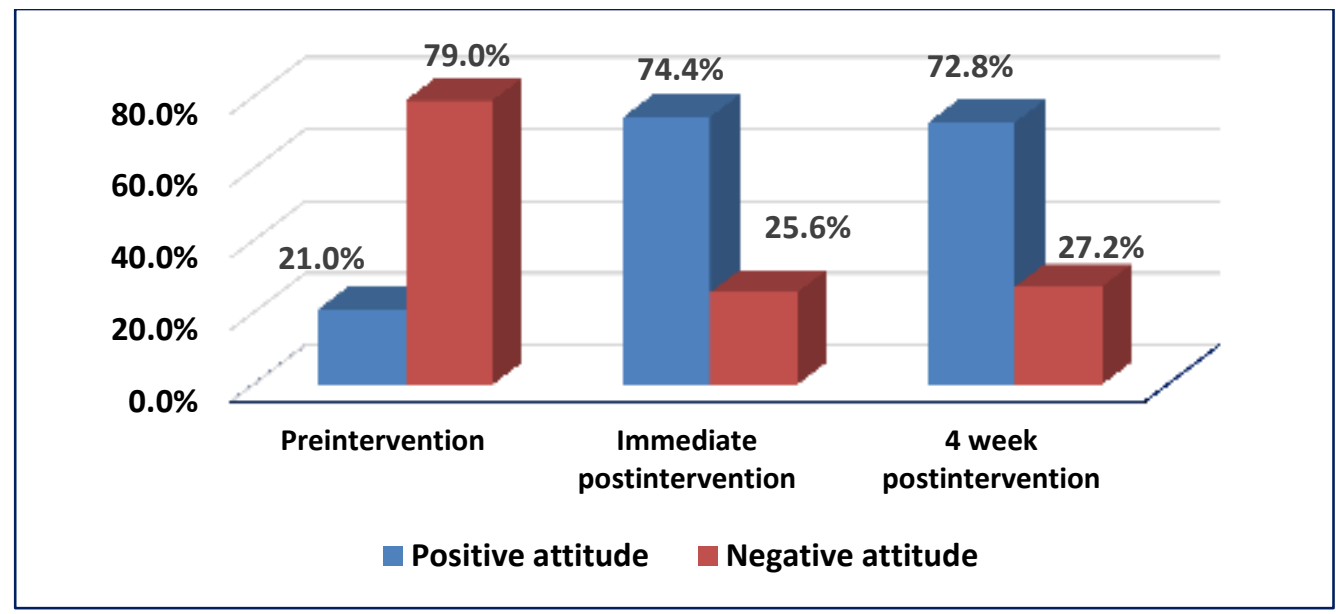

Figure (2): Percentage distribution of studied sample regarding their total attitude score at pre, immediate post intervention and 4 weeks post intervention phases $(n=195)$

Table (5): Mean and standard deviation of studied sample regarding their health belief model at pre ,immediate post intervention and 4 weeks post intervention phases $(n=195)$.

\begin{tabular}{|l|c|c|c|c|c|}
\hline \multirow{2}{*}{$\begin{array}{l}\text { Health Belief } \\
\text { Model Items }\end{array}$} & Pre-intervention & $\begin{array}{c}\text { Immediate Post- } \\
\text { intervention }\end{array}$ & $\begin{array}{c}\text { 4 weeks post- } \\
\text { intervention }\end{array}$ & \multicolumn{2}{|c|}{ ANOVA } \\
\cline { 2 - 6 } & Mean \pm SD & Mean \pm SD & Mean \pm SD & F & p-value \\
\hline $\begin{array}{l}\text { Total } \\
\text { Perceived } \\
\text { susceptibility }\end{array}$ & $4.08 \pm 5.30$ & $10.84 \pm 5.01$ & $10.77 \pm 5.04$ & 112.0 & 0.000 \\
\hline $\begin{array}{l}\text { Total } \\
\text { Perceived } \\
\text { severity }\end{array}$ & $4.03 \pm 5.23$ & $10.81 \pm 4.96$ & $10.66 \pm 4.95$ & 114.3 & 0.000 \\
\hline $\begin{array}{l}\text { Total } \\
\text { perceived } \\
\text { benefits }\end{array}$ & $3.49 \pm 4.49$ & $9.25 \pm 4.25$ & $9.13 \pm 4.22$ & 112.4 & 0.000 \\
\hline $\begin{array}{l}\text { Total } \\
\text { Perceived } \\
\text { barriers }\end{array}$ & $6.00 \pm 8.00$ & $16.78 \pm 7.76$ & $16.54 \pm 7.66$ & 121.1 & 0.000 \\
\hline $\begin{array}{l}\text { Total Cues to } \\
\text { action }\end{array}$ & $3.73 \pm 4.99$ & $10.83 \pm 4.80$ & $10.65 \pm 4.75$ & 135.3 & 0.000 \\
\hline $\begin{array}{l}\text { Total } \\
\text { Perceived } \\
\text { self-efficacy }\end{array}$ & $2.73 \pm 3.61$ & $7.68 \pm 3.43$ & $7.49 \pm 3.39$ & 126.3 & 0.000 \\
\hline
\end{tabular}


Effect of the Educational Package based on Health Belief Model on Nursing Students'

Knowledge and Attitude Regarding Human Papillomavirus and Cervical Cancer

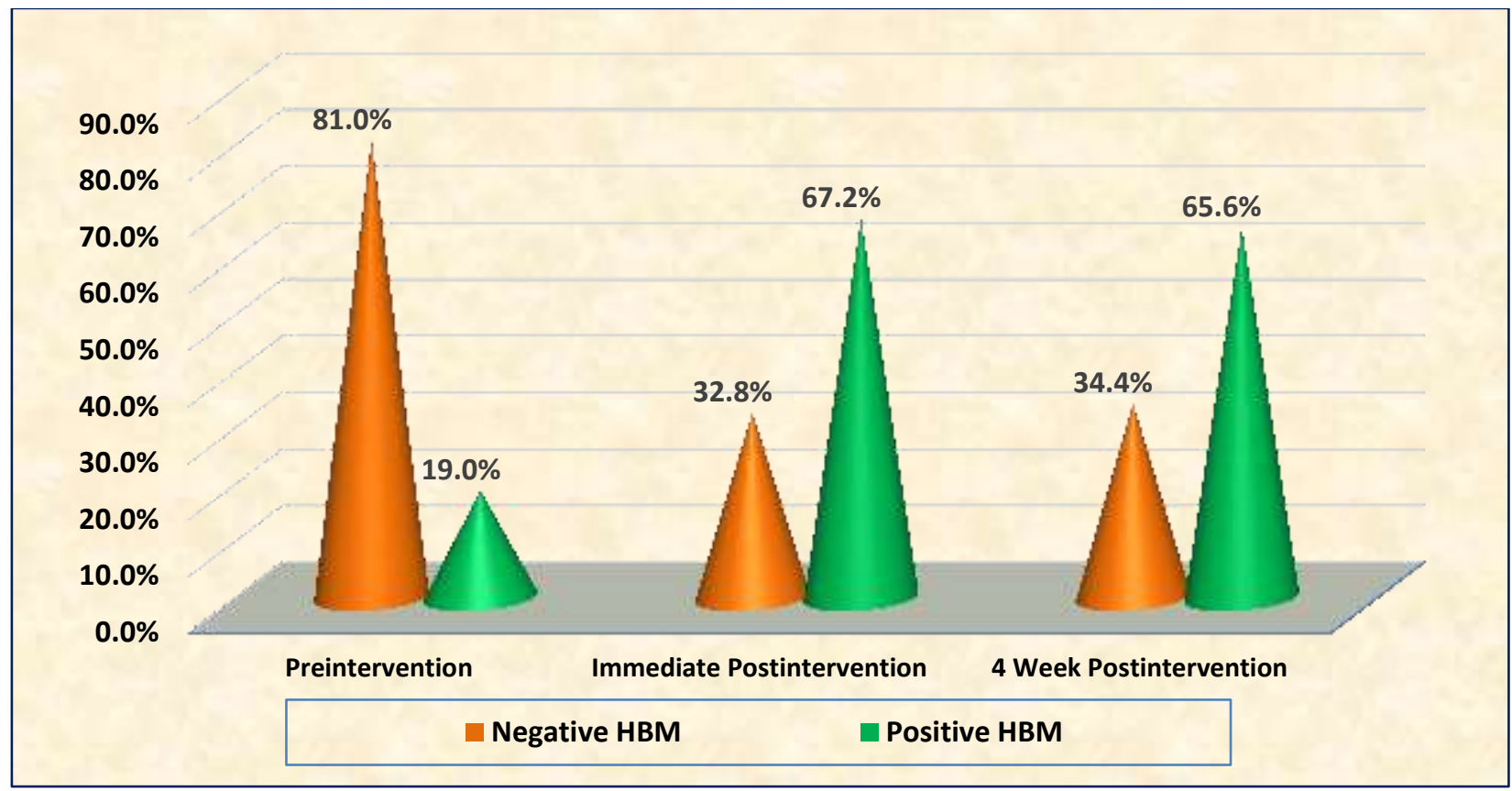

Figure (3): Frequency distribution of studied sample regarding total health belief model scores at pre, immediate post intervention and 4 weeks post intervention phases $(n=195)$

Table (6): Correlation coefficient between total knowledge, attitude and HBM scores at pre ,immediate post intervention and 4 weeks post intervention phases $(n=195)$.

\begin{tabular}{|c|c|c|c|c|c|c|c|}
\hline \multicolumn{2}{|c|}{$\begin{array}{l}\text { Total knowledge, } \\
\text { attitude and HBM }\end{array}$} & \multicolumn{2}{|c|}{ Pre-intervention } & \multicolumn{2}{|c|}{$\begin{array}{l}\text { Immediate Post- } \\
\text { intervention }\end{array}$} & \multicolumn{2}{|c|}{$\begin{array}{l}4 \text { Weeks Post- } \\
\text { intervention }\end{array}$} \\
\hline & & $\begin{array}{c}\text { Total } \\
\text { attitude }\end{array}$ & $\begin{array}{l}\text { Total } \\
\text { HBM }\end{array}$ & $\begin{array}{c}\text { Total } \\
\text { attitude }\end{array}$ & $\begin{array}{l}\text { Total } \\
\text { HBM }\end{array}$ & $\begin{array}{c}\text { Total } \\
\text { attitude }\end{array}$ & $\begin{array}{l}\text { Total } \\
\text { HBM }\end{array}$ \\
\hline \multirow{2}{*}{$\begin{array}{l}\text { Total } \\
\text { knowledge }\end{array}$} & $\mathrm{r}$ & 0.93 & 0.23 & 0.75 & 0.31 & 0.78 & 0.38 \\
\hline & p-value & $0.000 * *$ & $0.001 * *$ & $0.000 * *$ & $0.000 * *$ & $0.000 * *$ & $0.000 * *$ \\
\hline \multirow{2}{*}{$\begin{array}{l}\text { Total } \\
\text { attitude }\end{array}$} & $\mathrm{r}$ & & 0.25 & & 0.41 & & 0.48 \\
\hline & p-value & & $0.000 * *$ & & $0.000 * *$ & & $0.000 * *$ \\
\hline
\end{tabular}




\section{Discussion}

The aim of present study was to evaluate the effect of the educational package based on health belief model on nursing students' knowledge and attitude regarding human papilloma virus and cervical cancer. The current study result supported the stated hypothesis that Application of the educational package based on health belief model will have positive effect in increasing knowledge and improve positive attitude of nursing students regarding human papilloma virus and cervical cancer.

Regarding general characteristics of the studied nursing students, the current study results revealed that about more than three quarters of studied nursing students were in age group 18 years old with a mean age of $17.86 \pm 0.51$ years. This finding agreed with Atitt-allah, et al (2019) who conducted a study to evaluate "Effect of Educational Intervention on Knowledge and Attitudes Regarding Human Papillomavirus Infection and Its Vaccination among Nursing Students ", indicated that about three quarters of studied sample were in age group 17 years with a mean age of $17.86 \pm 0.551$ years.

As regards the residence, the finding of the present study clarified that about three quarters of the studied nursing students lived in rural areas, this may be due to geographical location where Benha university is the nearest to their residence .Concerning the marital status; the majority of them were single. This result matched with Atitt-allah, et al (2019) who indicated that approximately all of them were single.

Moreover; the results of current study revealed that all studied nursing students didn't attend any special scientific courses on cervical cancer and human papillomavirus.

This finding was in agreement with Eittah, et al (2020) who studied "Enhancing the Knowledge of Cervical Cancer Screening among Female Nursing Students: An
Interventional Educational Program" and the results showed that approximately all of students had never attend any previous educational activity regarding cervical cancer. Such similarity in the results can be attributed to the similarities in the properties of the studied samples.

Regarding nursing students' knowledge on cervical cancer, Pap smear test, human papillomavirus infection and human papillomavirus vaccination the present study revealed that there was a marked improvement regarding all items of studied sample total knowledge (knowledge about cervical cancer, pap smear test, human papillomavirus infection, and human papillomavirus vaccine) post implementation of the educational package based on health belief model with a highly statistical significant difference $(\mathrm{p}<0.001)$ between pre ,immediate post, and four weeks post intervention. This improvement in the students' knowledge might be due to students' active participation and good communication with the researcher who helped them to acquire knowledge.

This study results have been strengthened and enhanced by Barry and Amherst (2013) who studied "Increasing Knowledge about HPV and the HPV Vaccine Amongst Adolescents and Adults Through a School-Based Setting" illustrated that there was an increase in knowledge about HPV infection and the HPV vaccine after implementation of educational intervention.

These findings are in agreement with Ahmed, et al (2018) who conducted a study titled" Health Belief Model-based educational program about cervical cancer prevention on women knowledge and beliefs" reported that the mean score of knowledge before the intervention was low regarding cervical cancer, whereas after the educational intervention, the level of knowledge improved significantly. 


\section{Effect of the Educational Package based on Health Belief Model on Nursing Students' \\ Knowledge and Attitude Regarding Human Papillomavirus and Cervical Cancer}

Moreover, the results of current study are confirmed by Atitt-allah, et al (2019) who demonstrated that there was improvement in the level of all knowledge items regarding human papilloma virus infection and its vaccination, where the minority and the majority of studied sample had good knowledge regarding human papilloma virus infection and its vaccination at pre-intervention and post-intervention phases respectively.

This result matched with Eittah, et al (2020) who stated that at pre-test, the majority of the participants had poor knowledge about Pap smear test. However, the post-test showed great improvement in the students' knowledge regarding all of items of knowledge.

Finally, the above mentioned results can be concluded as there was a highly statistical significant difference between the results of immediate post-test and four weeks post intervention compared to pre-test in favor of post-test regarding all items of students' total knowledge regarding cervical cancer, PAP smear, human papilloma virus infection and its vaccination. So, there is a need for all stakeholders to step up awareness creation for cervical cancer, HPV infection and improved HPV vaccination through more educational programs, mass media or university and preuniversity curriculum.

\section{Concerning attitude of the studied nursing} students, the results of current study displays that, about, one fifth, three quarters and slightly less than three quarters of studied sample had positive total attitude score at pre-intervention, immediate post-intervention and four weeks post intervention phases respectively. On the other hand, it reveals that majority, one quarter and slightly more than one quarter of studied sample had negative total attitude score at preintervention, immediate post-intervention and four weeks post intervention phases respectively. Moreover, there is a marked improvement in all items of studied sample attitude regarding all items of students' attitude regarding cervical cancer, PAP test, and human papilloma virus infection and human papilloma virus vaccination post implementation of the educational package based on health belief model with a highly statistical significant difference $(\mathrm{p}<0.001)$ between pre, immediate post, and four weeks post intervention. This may be due to interventional sessions provided the participants with valuable information that can positively affect their beliefs which in turn positively affect their attitude.

The study findings were in the same line with Said, et al (2018) who studied "Effect of an Educational Intervention on Women's Knowledge and Attitude Regarding Cervical Cancer" who revealed that the studied sample attitude was highly positive after the implementation of the educational intervention. Furthermore, Kamada, et al (2018) who studied "What information can change the attitude toward the human papillomavirus vaccine, in Nagoya, Japan Society?", illustrated that most of the respondents did not know about the natural history of cervical cancer and Only more than one quarter believe that HPV is the cause of cervical cancer, although majority of them believe that HPV vaccine would prevent cervical cancer. Few respondents had positive attitude regarding adverse events following immunization.

Ultimately, it can be concluded that there was a highly statistical significant difference between the results of immediate post-test and four weeks post-test compared to pre-test regarding all items of students' total attitude regarding cervical cancer ,PAP smear test ,human papilloma virus infection and its vaccination. This can be explained by the fact that improved knowledge leads to a positive changing in attitudes and beliefs of studied sample. This also supports the necessary need 
for publishing health educational intervention especially regarding issues such as HPV which is mainly sexually transmitted disease and cervical cancer.

\section{Regarding the mean scores of} constructs of $\mathrm{HBM}$, the present study indicates that the nursing students immediately after and four weeks after the program got a highly significant difference scores regarding the perceived susceptibility, the perceived severity, the perceived barriers, the perceived benefits, the cues to action and perceived self -efficacy compared with the scores before the program with $(\mathrm{p}<0.001)$. This might be because of the identification of the severity and benefits. Moreover, the nursing educational program might increase the awareness and motivation of the students. These study findings supported that the educational package based on HBM will change health beliefs positively toward cervical cancer and HPV.

The mean scores of all items of health belief model were low before implementing the educational package and there was a highly statistical significant difference between the results of immediate post-test and four weeks post test compared to pre-test. This was in the same line with a study carried out on students in Faculties of Benha University by Yossif and EL Sayed (2014) who studied "Effect of selflearning package based on health belief model on cervical cancer prevention among female university students" found that, after implementation of self-learning package, there was a significant improvement in the mean scores of health belief model constructs.

Also, these results were in agreement with a study carried out by Ahmed, et al (2018) who revealed that education based on HBM was effective and improved all items of health belief model.

In relation to correlation between total knowledge and total attitude score, the findings of present study clarified that, there was a highly positive statistical correlation between total knowledge and total attitude regarding cervical cancer and human papilloma virus at pre and immediate post intervention and four weeks post-intervention phases. This can be attributed to the fact that improved knowledge is in turn improving attitudes and beliefs and vice versa, which underlines the importance of educational programs.

The result of current study was in agreement with Adesina, et al (2018) who studied "Knowledge, Practice and Acceptability of HPV Vaccine by Mothers of Adolescent Girls in Ilorin, Nigeria," stated that respondents with good knowledge of HPV and cervical cancer were more positive in their attitude and willing regarding cervical cancer and HPV than respondents with poor knowledge ( $\mathrm{P}<0.001)$.

On the other hand, this result came contrary to the result of Jeyachelvi, et al (2016) who studied "Human papillomavirus infection and its vaccines: knowledge and attitudes of primary health clinic nurses in Kelantan, Malaysia" illustrated that the participants have favorable attitudes towards HPV vaccination; however they have significant knowledge deficit and major misunderstanding in critical knowledge items. This dissimilarity of present study result with the result of this study could be due to different demographic characteristics, traditions and believes of the studied population.

The results of the current study indicated that there was a highly positive statistically significant correlation between total knowledge score and health belief Model constructs scores immediate post and four weeks after the program. This might be because of nursing educational program, as it provided the participants with valuable information that can affect their health beliefs and preventive behaviors. These findings are congruent with the study by Ahmed, et al (2018) who reported that there was a significant positive correlation 


\section{Effect of the Educational Package based on Health Belief Model on Nursing Students' \\ Knowledge and Attitude Regarding Human Papillomavirus and Cervical Cancer}

between total knowledge and total health beliefs scores.

\section{Conclusion}

Application of the educational package based on health belief model have a positive effect on improving nursing students' knowledge and attitude regarding human papilloma virus and cervical cancer . the mean total score of health belief model and its subscales, perceived susceptibility, perceived severity, perceived benefits, perceived barriers, self-efficacy and cues to action post- educational program are significantly higher immediate and four weeks post intervention compared to pre intervention. Finally, there was a statistically significant positive correlation between total knowledge, attitude and health belief scores pre intervention, immediate post intervention and four weeks post intervention. Therefore, the study hypothesis was supported and the aim was achieved.

\section{Recommendations}

*Reapplication of health belief model for all university students as a healthy preventive behavior towards human papilloma virus and cervical cancer.

*Integration of health belief model into the under graduate and post graduated student nurse at woman's health department, faculty of nursing, Benha university.

*Woman's health department must design and distribute brochures about early detection and screening of human papilloma virus and cervical cancer including pictures and diagrams to all women and nurses attending maternity department at benha university hospital.

\section{Further studies need to be performed:}

*Evaluate the effect of educational package based on HBM on women's health beliefs regarding human papilloma virus and cervical cancer.
*Future study should aim to conduct a similar study on a large sample for generalizing the findings.

*Further prospective research is needed concerning application health belief model regarding nurses' knowledge toward human papilloma virus and cervical cancer.

\section{References}

Adesina KT, Saka A, Isiaka-Lawal SA, Adesiyun OO, Gobir A, Olarinoye AO and Ezeoke GG. (2018). "Knowledge, Practice and Acceptability of HPV Vaccine by Mothers of Adolescent Girls in Ilorin, Nigeria," Sudan Journal of Medical Sciences, 13 (1): 33-49.

Ahmed, S. R. H., Esa, A. S. M., \& Mohamed El-zayat, O. S. (2018). Health Belief Modelbased educational program about cervical cancer prevention on women knowledge and beliefs. Egyptian Nursing Journal, 15(1): 3949.

Ampofo, A. G., Adumatta, A. D., Owusu, E., \& Awuviry-Newton, K. (2020). A crosssectional study of barriers to cervical cancer screening uptake in Ghana: An application of the health belief model. PloS one, 15(4): 231459.

Annan, F. M., Asante, K. O., \& Kugbey, N. (2019). Perceived seriousness mediates the influence of cervical cancer knowledge on screening practices among female university students in Ghana. BMC women's health, 19(1): 1-8.

Atitt-Allah, N. A. A. H., Abd-Elhady, R. M., \& Araby, O. A. W. A. (2019). Effect of Educational Intervention on Knowledge and Attitudes Regarding Human Papillomavirus Infection and Its Vaccination among Nursing Students. American Journal of Nursing Research, 7(4): 453-464.

Barry D and Amherst U. (2013). Increasing Knowledge about HPV and the HPV Vaccine Amongst Adolescents and Adults Through a School-Based Setting: A Capstone Project, 
Doctor of Nursing Practice (DNP) Projects. 31. University of Massachusetts Amherst, College of Nursing Retrieved from: https://scholarworks.umass.edu/nursing_dnp_c apstone/31. Accessed on january, 3 2021, 10 PM.

Chan, C. K., Aimagambetova, G., Ukybassova, T., Kongrtay, K., \& Azizan, A. (2019). Human papillomavirus infection and cervical cancer: epidemiology, screening, and vaccination-review of current perspectives. Journal of oncology, 45(7):8090.

Dönmez, S., Öztürk, R., Kısa, S., Karaoz Weller, B., \& Zeyneloğlu, S. (2019). Knowledge and perception of female nursing students about human papillomavirus (HPV), cervical cancer, and attitudes toward HPV vaccination. Journal of American College Health, 67(5): 410-417.

Eghbal, S. B., Karimy, M., Kasmaei, P., Roshan, Z. A., Valipour, R., \& Attari, S. M. (2020). Evaluating the effect of an educational program on increasing cervical cancer screening behavior among rural women in Guilan, Iran. BMC Women's Health, 20(1): 19.

Eittah, H. F. A., Aljohani, K. A. S., \& Aljohani, M. S. E. (2020). Enhancing the Knowledge of Cervical Cancer Screening among Female Nursing Students: An Interventional Educational Program. Sudan Journal of Medical Sciences (SJMS), pp 431439.

Hampson, I. N., Oliver, A. W., \& Hampson, L. (2021). Potential Effects of Human Papillomavirus Type Substitution, Superinfection Exclusion and Latency on the Efficacy of the Current L1 Prophylactic Vaccines. Viruses, 13(1):p 22.

Jeyachelvi K, Juwita $S$ and Norwati $D$. (2016). Knowledge and Attitudes of Primary Health Clinic Nurses in Kelantan, Malaysia, Asian Pac J Cancer Prev, 17 (8): 3983-3988.
Kamada M, Inui H, Kagawa T, Mineda A, Tamura T, Fujioka T, Motoki T, Hirai H, Ishii $E$ and Irahara M. (2018). What information can change the attitude toward the human papillomavirus vaccine, in Nagoya, Japan Society?" J. Obstet. Gynaecol. Res. 44(4): 778-787

Maver, P. J., \& Poljak, M. (2019). Primary HPV-based cervical cancer screening in Europe: implementation status, challenges, and future plans. Clinical Microbiology and Infection, 26(66):89-99

Naz, M. S. G., Kariman, N., Ebadi, A., Ozgoli, G., Ghasemi, V., \& Fakari, F. R. (2018). Educational interventions for Cervical Cancer Screening Behavior of Women: a systematic review, 6(5):13-16.

Said, S., Hassan, H., \& Sarhan, A. (2018). Effect of an Educational Intervention on Women's Knowledge and Attitude Regarding Cervical Cancer. American Journal of Nursing Research, 6(2): 59-66.

Yossif HA, EL Sayed HA (2014). Effect of self-learning package based on health belief model on cervical cancer prevention among female university students. IOSR J Nurs Health Sci 3(1):77-88 


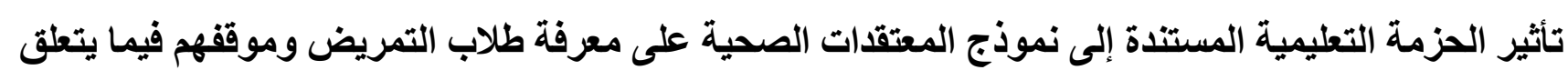

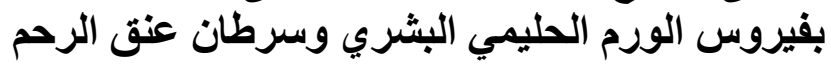

عزيزة إبراهيم محمود، سامية عبد الحكيم حسنين عبود ، إيمان محم عبد الحكم ، فاطمة كمال علي

يعتبر سرطان عنق الرحم مشكلة صحية كبيرة في جميع أنحاء العالم حيث أنه ثاني أكثر أنواع السرطانات شيوعًا بين النساء على مستوى العالم. لذا هدفت هذه الدراسة إلى تقييم تأثير الحزمة التعليمية بناءً على نموذج الاعتقاد الصحي على معرفة طلاب التمريض وموققهم تجاه فيروس الورم الحليمي البشري وسرطان عنق الرحم. وقد أجريت الدراسة بكلية التمريض بجامعة بنها. على 190 طالبة تمريض. وقد خلصت الدراسة بأن تطبيق الحزمة التعليمية على أساس نموذج المعتقد الصحي له تأثير إيجابي على تحسين معرفة طلاب التمريض وموقفهم تجاه فيروس الورم الحليمي البشري وسرطان عنق الرحم. كما اوصت الدراسة بتطبيق نموذج المعتقد الصحي لجميع طلاب الجامعة كسلوك وقائي صحي تجاه فيروس الورم الحليمي البشري وسرطان عنق الرحم. 